\title{
Necrotising otitis externa: an unusual cause of cranial nerve palsy in a diabetic haemodialysis patient
}

\author{
${ }^{1} \mathrm{ACM}$ Thompson, ${ }^{2} \mathrm{I}$ Zammit-Maempel, ${ }^{3} \mathrm{~F}$ Stafford, ${ }^{4} \mathrm{M}$ Narayanan, ${ }^{5} \mathrm{NS}$ Kanagasundaram \\ ${ }^{1}$ Specialist Trainee, Core Medical Training, Northern Deanery; ${ }^{2}$ Consultant Radiologist; ${ }^{3}$ Consultant Ear, Nose, Throat; ${ }^{4}$ Consultant \\ Microbiologist; ${ }^{5}$ Consultant in Renal Medicine, Newcastle upon Tyne Hospitals NHS Foundation Trust, UK
}

ABSTRACT We present an unusual case of necrotising otitis externa (NOE) causing a lower motor neurone facial nerve palsy in a patient with diabetes mellitus and receiving maintenance haemodialysis for end-stage renal disease (ESRD). Pseudomonas aeruginosa is the most common pathogen isolated in NOE, although our case involved the non-typical pathogens Aspergillus flavus and Proteus mirabilis. We discuss the need for diagnostic rigour and the importance of considering atypical infective pathology in patients with ESRD or diabetes mellitus. We review NOE with reference to causative agents, imaging strategies, prognostic indicators and treatment.

KEYWORDS Aspergillus flavus, diabetes mellitus, haemodialysis, malignant otitis externa, necrotising otitis externa, Proteus mirabilis, Pseudomonas aeruginosa
Correspondence to AC Thompson, Department of Renal Medicine, Freeman Hospital, High Heaton, Newcastle upon Tyne NE7 7DN, UK

tel. +44 (0)191 2336161

e-mail acmthompson@doctors.org.uk

DECLARATION OF INTERESTS No conflict of interests declared.

\section{CASE REPORT}

A 77-year-old South Asian man presented to the acute admissions service with a one-month history of progressive, left-sided facial weakness and left-sided mandibular and pre-auricular pain. A diagnosis of Bell's palsy was made. He was receiving ongoing treatment for ipsilateral otitis externa with topical tri-adcortyl ointment. His medical history included end-stage renal disease (ESRD) of uncertain cause, treated with hospital haemodialysis; poorly controlled insulin-treated type 2 diabetes mellitus; ischaemic heart disease; and gout.

Unfortunately, the patient's symptoms continued to deteriorate and he was admitted to renal services from the haemodialysis unit. On examination he had periauricular tenderness and purulent discharge from the left auditory canal and associated conductive hearing loss. There was a left lower motor neurone facial palsy, with grade III upper division involvement and grade IV lower division involvement (House-Brackmann classification).' Other cranial nerves were spared. Inflammatory markers were mildly elevated with a C-reactive protein of $7 \mathrm{I} \mathrm{mg/l.} \mathrm{Glycosylated}$ haemoglobin $\left(\mathrm{HbA}_{\mathrm{lc}}\right)$ of $10.7 \%$ reflected recent poor glycaemic control. Left-sided otoscopy revealed posteroinferior perforations of the tympanic membrane, with debris and mucopurulent discharge on the left, and pure-tone audiometry confirmed conductive deafness. A contrast-enhanced computerised tomography (CT) scan demonstrated soft tissue thickening in the external auditory canal and a $2.7 \times 1.3 \mathrm{~cm}$ mass below the left skull base, engulfing the carotid artery and severely compressing the internal jugular vein. The mass extended through the parapharyngeal space onto the lateral pterygoid and deep temporalis muscles (Figure I). Auricular swabs grew Aspergillus flavus and Proteus mirabilis.
A diagnosis of necrotising (malignant) otitis externa (NOE) was made. The patient was treated for a total of six weeks with systemic agents and localised intraauricular ribbon gauze. He completed four weeks of intravenous meropenem (I g once daily), combined with oral ciprofloxacin ( $250 \mathrm{mg}$ twice daily) and voriconazole (400 mg mane, $200 \mathrm{mg}$ nocte). Thereafter he continued with a further two weeks of oral ciprofloxacin and voriconazole. Doses were adjusted for the renal impairment and haemodialysis requirements. Topical ribbon gauze soaked with amphotericin and nystatin was administered daily over this period. The initial treatment was based upon the likelihood that Pseudomonas is the usual causative agent, and that resistant strains of Pseudomonas are increasingly prevalent. Culture results guided treatment thereafter.

Two months later the facial nerve palsy was improving so that the lower division involvement had reduced to grade II weakness. Otoscopy confirmed reduced suppurative discharge with ongoing dry perforation of the tympanic membrane. Follow-up CT scans at three months and then seven months from diagnosis showed continuing improvement (Figure 2).

\section{DISCUSSION}

This case highlights a number of key learning points. The ESRD and diabetic populations have increased susceptibility to infection. ${ }^{2.3}$ According to the UK Renal Registry, infection was the second most common cause of death in a population on renal replacement therapy between 2000 and 2006. ${ }^{4}$ In this group infection accounted for nearly $20 \%$ of mortality, and was second only to cardiac disease. An infective aetiology should be considered in any unusual presentation in this population. 


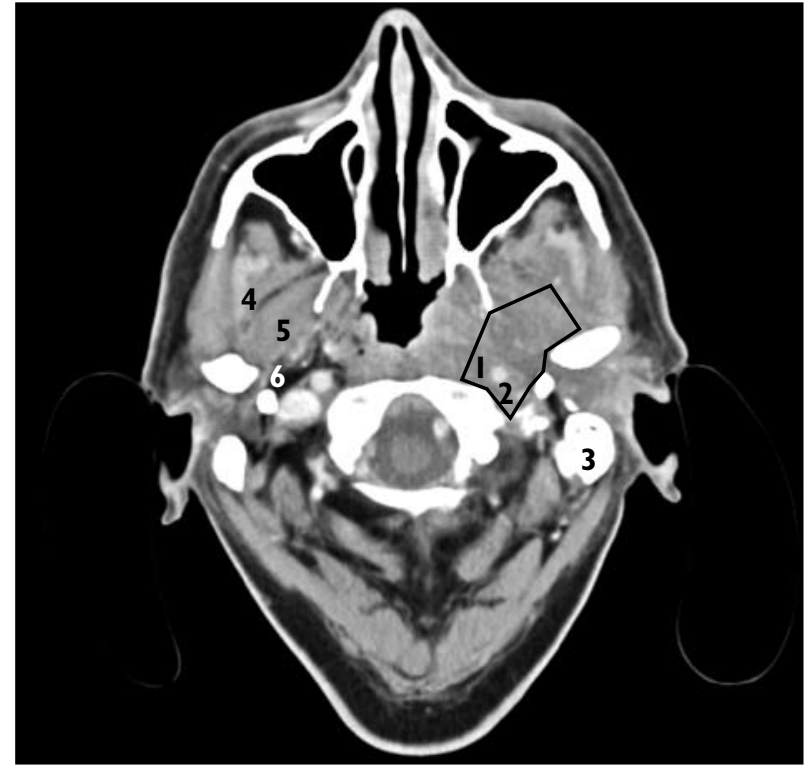

FIGURE I Computed tomography image at the time of diagnosis. A $2.7 \times 1.3 \mathrm{~cm}$ mass (marked with black line) below the left skull base engulfs the carotid artery and severely compresses the internal jugular vein. The mass extends through the parapharyngeal space onto the lateral pterygoid and deep temporalis muscles. (I. Carotid artery; 2. Internal jugular vein; 3. Mastoid; 4. Deep temporalis muscle; 5. Lateral pterygoid; 6. Parapharyngeal space)

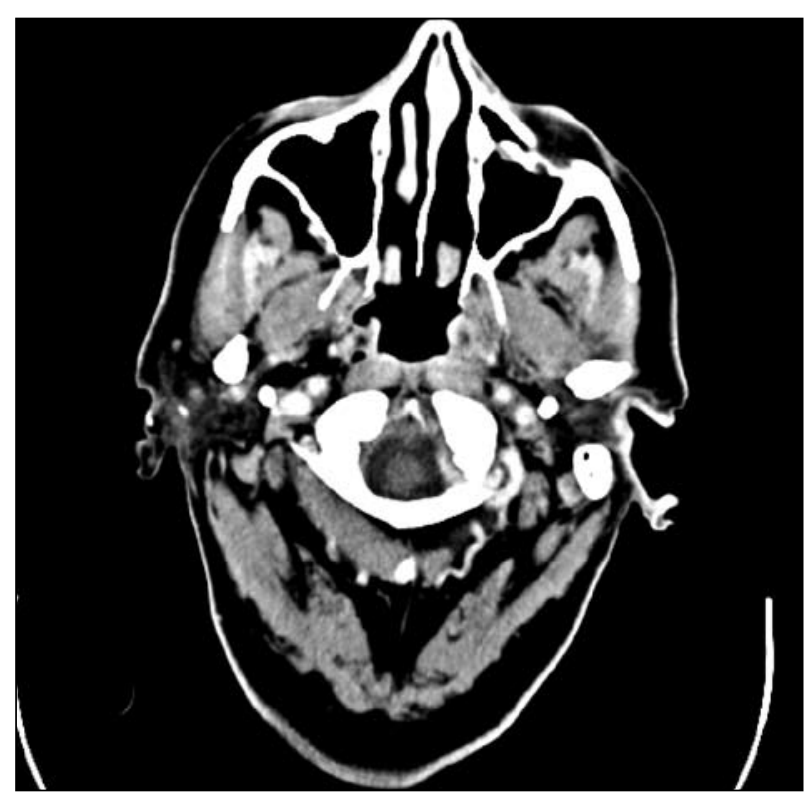

FIGURE 2 Follow-up CT scan seven months later. The cuff of tissue around the left carotid artery, internal jugular vein and soft tissue around the lateral pterygoid is less marked than previously.

In our case the symptoms of otalgia and discharge should have raised the suspicion of localised infection or malignancy, mandating otoscopy. Particularly important otoscopic features would include granulation in the bone-cartilage junction of the external auditory canal as one of the hallmarks of NOE. ${ }^{5}$ Bell's palsy should be a diagnosis of exclusion and poor response to treatment should raise the possibility of misdiagnosis or an atypical infective pathology.
Necrotising otitis externa is more accurately described as skull base osteomyelitis. In the past it has been defined by the diagnostic criteria of Cohen and Friedman; 6 however, these criteria are not well established, particularly with regards to imaging strategies, and are no longer strictly applied when the clinical scenario is suggestive. Diabetes mellitus as a causative agent is described in these diagnostic criteria, but any form of immunosuppression would be a predisposing factor and this should be recognised. Endstage renal disease and haemodialysis as specific causative factors have only been described in two case studies in the literature to our knowledge. ${ }^{78}$ In our case the combination of factors conveying immunosuppression were contributory to the presentation of NOE.

Pseudomonas aeruginosa is isolated from the external auditory canal in more than $90 \%$ of cases with NOE. ${ }^{9}$ Case reports identify the minority of other causative agents, including Staphylococcus aureus, ${ }^{10}$ Staphylococcus epidermidis, ${ }^{\prime \prime}$ Klebsiella, ${ }^{12}$ Proteus mirabilis ${ }^{13}$ and various Aspergillus and Candida species. ${ }^{714-16}$ In this case, infection was as a result of Aspergillus flavus and Proteus mirabilis, with no evidence of Pseudomonas on repeated culture. Assumptions that Pseudomonas is the causative pathogen can lead to a delay in appropriate treatment. Increasing numbers of ciprofloxacin-resistant Pseudomonas infections means that identifying antibiotic sensitivity remains important. ${ }^{17}$

Mortality data in NOE is poorly documented, but fatal events are usually due to intracranial complications such as dural sinus thrombosis, meningitis and intracranial abscesses. ${ }^{5,9} \mathrm{~A}$ recent case series of 46 patients treated for NOE quoted healing rates over $95 \%$ after a mean follow-up of around 78 weeks. ${ }^{18}$ Cranial nerve palsy as a complication of NOE is well recognised, but debate remains about whether this feature has prognostic value. The facial nerve is most commonly affected because of the close relationship of the stylomastoid foramen to the external auditory canal. Facial nerve palsy is associated with more advanced disease on CT imaging and is also less likely to improve with medical treatment when compared with other cranial nerve palsies. However, higher rates of mortality have not been demonstrated. ${ }^{18-20}$

Temporomandibular joint (TMJ) involvement is rare and may reflect more advanced disease with worse prognosis. Data are limited: one case series of 42 people with NOE identified six patients with TMJ involvement, and reported a $50 \%$ mortality rate directly related to the infection within the TMJ group. ${ }^{21}$

No single imaging strategy is universally effective for both diagnosis and follow-up of NOE.22 The Cohen-Friedman criteria advocates technitium 99 (Tc99) scintigraphy bone scanning as first-line imaging, but this provides poor anatomic detail, changes are not specific for infective infiltration and a soft tissue mass without bone involvement will not be detected. Instead, many units now use CT 
imaging alone for a quick and effective initial assessment of the extent of spread. Magnetic resonance imaging (MRI) can give additional information about soft tissue changes, bone marrow infiltration and intracranial involvement, but is more time consuming and has less sensitivity to detect subtle bony erosion. Computed tomography, MRI and Tc99 scintigraphy are all limited in determining disease resolution as changes on CT and MRI will persist for a time following effective treatment, and Tc99 scans remain positive until osteoblastic activity has ceased. Disease resolution can be assessed with gallium imaging as gallium binds with bacteria and lactoferrin from leucocytes, making this sensitive and specific for detecting soft tissue and bone inflammation secondary to infection. However, it is expensive and the restricted anatomic detail makes this imaging less effective in the initial imaging assessment. In practice, several centres use CT or MRI at least three months after commencing treatment to evaluate response.

Long-term antimicrobials are the mainstay of treatment. Initially, the advent of quinolones made treatment of Pseudomonas infections amenable to outpatient oral antibiotic regimes. However, ciprofloxacin resistance has led to the increasing use of intravenous alternatives. Cautious prescribing with regards to nephrotoxicity, drug elimination and efficacy following dialysis is particularly important in cases of ESRD. Surgery is rarely used but may have a limited role for biopsy, local debridement and abscess drainage. Hyperbaric oxygen is sometimes used in treatment-resistant cases, but a Cochrane review in 2005 concluded that there was insufficient evidence for its use. ${ }^{23}$

\section{CONCLUSION}

Necrotising otitis externa is a rare but important cause of lower motor neurone facial palsy that can result in mortality from intracranial complications. People with diabetes mellitus or those who are immunocompromised are particularly susceptible, and our case highlights the importance of diagnostic rigour in this group of patients. Otoscopy should be performed early to look for granulation in the bone-cartilage junction, and imaging should be guided by the clinical question, taking into account differing diagnostic and monitoring needs. Early identification of the causative pathogen and resistance patterns is vital to allow appropriate antibiotic treatment, and long-term antimicrobials are the mainstay of treatment. Poor response to treatment should always alert the physician to reconsider the underlying diagnosis or causative agent.

\section{REFERENCES}

I House JW, Brackmann DE. Facial nerve grading system. Otolaryngol Head Neck Surg 1985; 93:146-7.

2 Kato $\mathrm{S}$, Chmielewski M, Honda $\mathrm{H}$ et al. Aspects of immune dysfunction in end-stage renal disease. Clin J Am Soc Nephrol 2008; 3:1526-33. doi: 10.2215/CJN.00950208

3 Vanholder R, Van Loo A, Dhondt AM et al. Influence of uraemia and haemodialysis on host defence and infection. Nephrol Dial Transplant 1996; 1 1:593-8.

4 Renal Association, UK Renal Registry. The eleventh annual report Bristol: UK Renal Registry; 2008. Available from: http://www. renalreg.com

5 Handzel O, Halperin D. Necrotizing (malignant) external otitis. Am Fam Physician 2003; 68:309-12.

6 Cohen D, Friedman P. The diagnostic criteria of malignant external otitis. J Laryngol Otol 1987; 101:216-21. doi:10.1017/ $\$ 0022215100101562$

7 Bae WK, Lee KS, Park JW et al. A case of malignant otitis externa caused by Candida glabrata in a patient receiving haemodialysis. Scand J Infect Dis 2007; 39:370-2. doi:10.1080/00365540600978971

8 Mitchell C,Weston M, Parsons V et al. 'Malignant' otitis externa in a patient on haemodialysis. Br J Clin Pract 1975; 29:126-7.

9 Rubin Grandis J, Branstetter BF, Yu VL. The changing face of malignant (necrotising) external otitis: clinical, radiological, and anatomic correlations. Lancet Infect Dis 2004; 4:34-9. doi: I0.1016/ SI 473-3099(03)00858-2

10 Keay DG,Murray JA. Malignant otitis externa due to Staphylococcus infection. J Laryngol Otol 1988; 102:926-7. doi:10.1017/ S0022215100106826

II Soldati D, Mudry A, Monnier P. Necrotizing otitis externa caused by Staphylococcus epidermidis. Eur Arch Otorhinolaryngol 1999; 256:439-4I. doi:I0.1007/s004050050184

12 Yang TH, Kuo ST,Young YH. Necrotizing external otitis in a patient caused by Klebsiella pneumoniae. Eur Arch Otorhinolaryngol 2006; 263:344-6. doi:10.1007/s00405-005-0998-y

13 Coser PL, Stamm AE, Lobo RC et al. Malignant external otitis in infants. Laryngoscope 1980; 90:312-6.

14 Cunningham M,YuVL, Turner J et al. Necrotizing otitis externa due to Aspergillus in an immunocompetent patient. Arch Otolaryngol Head Neck Surg 1988; I 14:554-6.

I5 Mani R, Belcadhi M, Krifa N et al. [Fungal necrotising external otitis.] Ann Otolaryngol Chir Cervicofac 2008; 125:40-5. In French. doi:I0.1016/j.aorl.2007.06.003

16 Bellini C,Antonini P, Ermanni S et al. Malignant otitis externa due to Aspergillus niger. Scand J Infect Dis 2003; 35:284-8. doi:I0.1080/00365540310000247

17 Berenholz L, Katzenell U, Harell M. Evolving resistant pseudomonas to ciprofloxacin in malignant otitis externa. Laryngoscope 2002; I 12:1619-22. doi:10.1097/00005537-200209000-00017

18 Franco-Vidal V, Blanchet $\mathrm{H}$, Bebear $\mathrm{C}$ et al. Necrotizing external otitis: a report of 46 cases. Otol Neurotol 2007; 28:77I-3. doi:10.1097/MAO.0b013e31805I53bd

19 Soudry E, Joshua BZ, Sulkes J et al. Characteristics and prognosis of malignant external otitis with facial paralysis. Arch Otolaryngol Head Neck Surg 2007; I33:1002-4. doi:I0.100 I/archotol.I33.10.1002

20 Mani N, Sudhoff H, Rajagopal S et al. Cranial nerve involvement in malignant otitis externa: implications for clinical outcome. Laryngoscope 2007; I 17:907-10. doi:10.1097/MLG.0b013e318039b30f

21 Mardinger O, Rosen D, Minkow B et al. Temporomandibular joint involvement in malignant external otitis. Oral Surg Oral Med Oral Pathol Oral Radiol Endod 2003; 96:398-403. doi:I0.1016/SI079. 2104(03)0047I-2

22 Okpala NC, Siraj QH, Nilssen E et al. Radiological and radionuclide investigation of malignant otitis externa. J Laryngol Otol 2005; I 19:7I-5. doi:I0.1258/00222I5053222978

23 Phillips JS, Jones SE. Hyperbaric oxygen as an adjuvant treatment for malignant otitis externa. Cochrane Database Syst Rev 2005; 2:CD0046I7. 